\title{
Laryngeal Initiation of Swallowing
}

\author{
Arthur T. Storey ${ }^{1}$ \\ Departments of Orthodontics and Physiology, Unizersity of Michigan, \\ Ann Arbor, Michigan
}

Received September 22; rezision received October 30, 1967

\begin{abstract}
A wide range of mechanical, chemical and thermal stimuli were applied to the epiglottis and larynx of decerebrate cats to determine the adequate stimulus (or stimuli) and most responsive sites for swallowing. Water was the most effective stimulus in eliciting swallowing from epiglottis, glottis and internal surface of the larynx. The glottis was the most effective site for the initiation of the reflex. The initiation of swallowing from the epiglottis and larynx is water specific; it is not due to mechanical or osmotic effects. The water effect is facilitated, however, by flow of the water over the receptive fields. Swallowing initiated from sites innervated by the superior laryngeal nerve may serve to guard the larynx from invasion by saliva and liquid bolus residucs or clear the larynx of secretions wafted up from the trachea and bronchi.
\end{abstract}

\section{Introduction}

The initiation of reflex swallowing, which shows considerable species variability, has been demonstrated by mechanical stimulation of the faucial pillars $(4,5)$, soft palate $(1,4,8,9)$, dorsal pharyngeal wall $(1,4,5)$, pharyngoesophageal junction (6), dorsum of the tongue (4), pharyngeal surface of the epiglottis $(1,6,9)$, internal surface of the larynx $(1,9)$; and by water stimulation of the soft palate (6), dorsal pharyngeal wall $(1,4$, 6 ), dorsum of the tongue (4), pharyngeal surface of the epiglottis (1), and the glossoepiglottidial sinuses (4). These sites of swallow elicitation are innervated by the trigeminal, glossopharyngeal, and superior laryngeal nerves. Since electrical stimulation of the superior laryngeal nerve is usually the most effective artificial means of eliciting swallowing, the epiglottis, the glottis, and the internal surface of the larynx-regions innervated by this nerve-might be expected to play a major role in the initiation of swallowing.

1 This study was supported in part by grant AM-07789 from the National Institute of Arthritis and Metabolic Diseases, National Institutes of Health, United States Public Health Service.

The author is indebted to Dr. Robert W. Doty for valued counsel and encouragement. His present address is Faculty of Dentistry, University of Toronto, 124 Edward Street, Toronto 2, Canada. 


\section{Method}

In the first experiment, 30 decerebrate cats, concomitantly used for single-unit studies of laryngeal receptors (7), had the larynx surgically exposed from the ventral aspect, divided in the midline and laid open with spring clips. All operations were conducted under surgical anesthesia with sodium amytal (Surital). Motor responses were visually noted following the application of light and heavy pressure, air movements, hot and cold isotonic saline solutions, distilled water and aqueous solutions of sodium chloride, sucrose, quinine hydrochloride, acetic acid, and alcohol in various concentrations to the exposed interior of the larynx, glottis, and laryngeal surface of the epiglottis.

Five other decerebrate cats were used in a second experiment to more precisely determine the mode of excitation and the relative significance of glottic stimulation compared to epiglottic stimulation. Isotonic saline and distilled water were applied restrictively to the glottis of the intact larynx by means of an intranasal polyethylene catheter or dropper in prone or supine animals. The epiglottis was excised in two animals to completely eliminate the possibility of epiglottic stimulation. The motor responses to applications of $0.1-0.4 \mathrm{ml}$ were monitored electromyographically. Two electrodes of 34-gauge enamel-nichrome wire (Driver Harris) were inserted by a means of 26-gauge hypodermic needles into the ipsilateral mylohyoid, thyroarytenoid, and inferior constrictor muscles and occasionally into an intercostal muscle after the technique of Inman (unpublished results) ${ }^{2}$. Electrode placements were verified at the termination of the experiment. Muscle-action potentials were displayed on a cathode-ray oscilloscope; one muscle (usually the mylohyoid) was monitored on a loud speaker. Pressure changes within the airway were monitored by means of a pressure transducer (Grass-PT 5) connected to a plastic cannula in the tracheal wall in the manner of Kawasaki, et al. (2) and displayed on the cathode-ray oscilloscope. Selected sequences were recorded on $35-\mathrm{mm}$ photographic paper for positive identification of the motor responses. Swallowing was identified by sequential activity in the mylohyoid, thyroarytenoid and inferior constrictor muscles, an absence of activity in the intercostal muscles, and no rise in intratracheal pressure. Coughing was characterized by synchronous activity in the thyroarytenoid and intercostal muscles, an absence of activity in the mylohyoid and inferior constrictor and a marked rise in intratracheal pressure. Typical records of swallowing and coughing are shown in Fig. 1. The time from application of the stimulus to each swallow was measured by stop watch to the nearest second.

2 The barbed wire electrode was developed by the Prosthetic Devices Research Project (now Biomechanics Laboratory) at the University of California, Berkeley and San Francisco, during studies of human locomotion. 


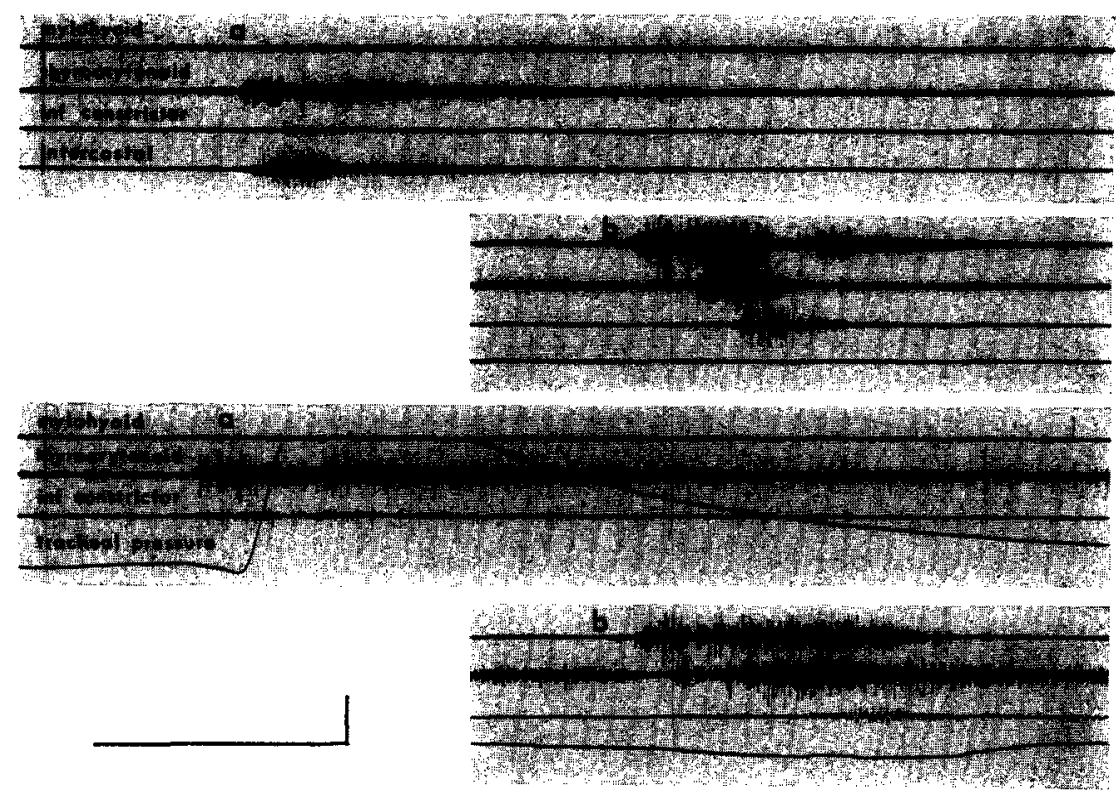

Fig. 1. A cough (a) followed by a swallow (b) in two cats. In the upper record coughing was elicited with water and in the lower record by mechanical stimulation. Synchronous activity in the thyroarytenoid and intercostal muscles and a marked rise in tracheal pressure characterizes a cough. Sequential activity in the mylohyoid, thyroarytenoid and inferior constrictor muscles with little change in tracheal pressure identifies a swallow. Vertical calibration represent $1 \mathrm{mV}$ for the intercostal muscle, $2.5 \mathrm{mV}$ for the thyroarytenoids, inferior constrictors and mylohyoid in the upper record, $5 \mathrm{mV}$ for the mylohyoid in the lower record and $10 \mathrm{~cm} \mathrm{H}_{2} \mathrm{O}$ for tracheal pressure. The horizontal calibration represents $0.5 \mathrm{sec}$. Approximately $1 \mathrm{sec}$ of the recording has been removed between the cough and the swallow in the upper record.

Stimulation was repeated $15-45$ times after residual excitation in the muscles had disappeared.

\section{Results}

In the first experiment hypotonic solutions and water produced swallowing when applied by dropper to the laryngeal surface of the epiglottis, glottis, and internal surface of the larynx. Isotonic and hypertonic solutions were much less effective than hypotonic solutions. Isotonic sucrose solution was as effective as water in initiating swallowing. Mechanical stimulation with a small ox-hair brush or plastic probe invariably produced glottic tensing usually followed by coughing. Swallowing rarely ensued from mechanical stimulation. The glottis and the base of the epiglottis were the most effective areas for eliciting these reflexes.

In the second experiment, in which the stimulus was restricted to the glottic area, water readily produced swallowing. Table 1 illustrates the 


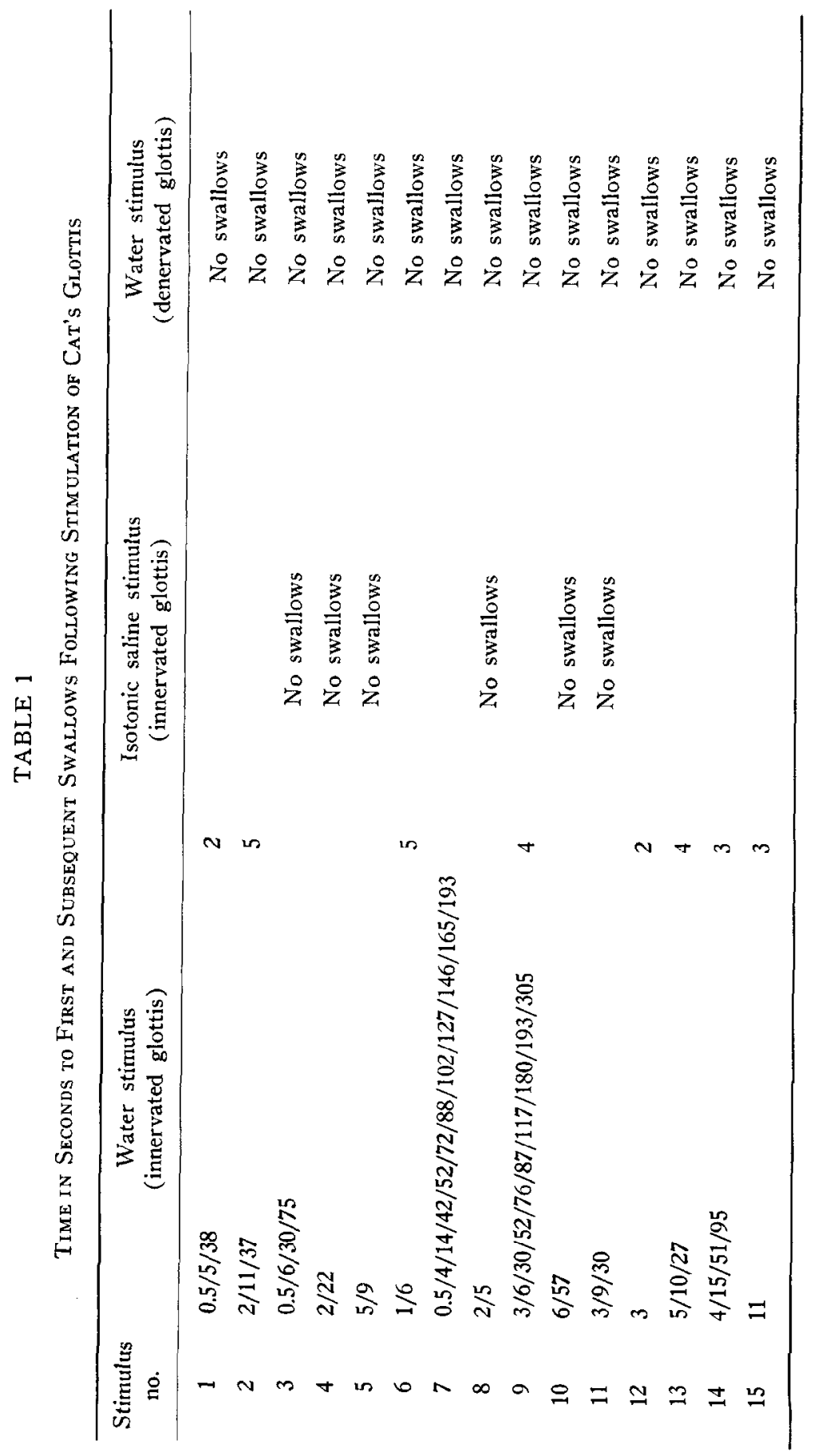


lively response of a prone cat, with the epiglottis resected, to the first 15 applications of $0.1 \mathrm{ml}$ of water injected through an intranasal catheter. Repetitive swallowing was characteristic of the best preparations in the early phases of stimulation. Spontaneous swallowing occurred periodically in supine animals but rarely in prone animals. Injection of more than 0.4 $\mathrm{ml}$ of water would occasionally result in coughing, particularly in cats in the supine position.

Swallowing usually terminated such coughing as shown in Fig. 1. Isotonic saline was much less effective in initiating swallowing as can be seen from Table 1. Severing both right and left superior laryngeal nerves (Table 1) completely abolished swallowing to water stimulation of the larynx. Swallowing occurred as readily in the two animals with the epiglottis excised as in cats with the epiglottis intact.

\section{Discussion}

Most of the studies on the initiation of swallowing from sites innervated by the vagus nerve, and specifically by its superior laryngeal branch, have implicated the pharyngeal surface of the epiglottis or the glossoepiglottidean sinuses $(1,4,6,9)$. The study of Miller and Sherrington (4) suggested that the epiglottis was not necessary for initiation of the reflex with water stimulation. Water poured on the dorsum of the tongue (which would flow into the glossoepiglottidean sinuses) in a preparation in which the epiglottis was excised swallowed "quite efficiently." Controlled application of water onto the glottis in this study, clearly identified the glottis itself as a very effective site for the initiation of swallowing in the cat. As in the elicitation of the reflex from the glossoepiglottidean sinuses (4) the temperature of the water is of little importance. This is the first evidence of swallowing initiated from the glottis to water stimulation. Kahn (1) and Waller and Prévost (9) had previously reported initiation of swallowing by mechanical stimulation of the glottis in the monkey and rabbit. Kahn (1) was unable to elicit swallowing by mechanical stimulation of the interior of the larynx in rabbits; swallowing which occurred was always associated with respiratory reflexes. Swallowing was very rarely initiated by mechanical stimulation of the interior of the larynx or glottis of the cat; swallowing frequently ensued after coughing as illustrated in Fig. 1. Swallowing initiated by mechanical stimulation of the glottis may well depend on the method of stimulation-stroking with a brush was more likely to elicit the reflex from the cat's larynx than pressure applied with a rigid probe. Although the role of mechanical stimulation is not well defined, it is very clear that water was an effective stimulus and that the glottis as well as the epiglottis is a potent reflexogenic site for swallowing

The adequate stimulus for swallowing seems to be chemical rather than 
mechanical for the responsiveness of the animal was markedly reduced when isotonic saline solution was substituted for water. Since the isotonic saline solution was applied in identical fashion to the water the initiation cannot be attributed to either impact or flow. Miller and Sherrington (4) were of the opinion that the fluidity of the water was of key importance in initiating the reflex. They found, however, that mercury allowed to run into a glossoepiglottidean sinus did not excite as readily as water, a larger quantity was required and the latency of the response was longer. Flow of the water may facilitate the chemical effect in the larynx, however. Rapid, rather than slow, injection of an equal volume of water onto the glottis shortened the latency of swallowing and resulted in a greater number of swallows.

Water elicitation of swallowing does not appear to be due to an osmotic effect. Isotonic sucrose solution was as effective as water in eliciting the reflex. A $20 \%$ solution of ethyl alcohol and water was equally or more effective than water alone in initiating swallowing, a finding similar to that of Miller and Sherrington (4).

Although many of the sites giving rise to swallowing may be excited by mechanical or water stimulation it appears that the reflex arises from mechanical stimulation at pharyngeal and oral sites. Kahn (1) suggested many years ago that swallowing initiated from sites innervated by the trigeminal nerve of the rabbit served an alimentary function and swallowing initiated from sites innervated by the glossopharyngeal and vagus nerves served a follow-up or protective function. Swallowing initiated from epiglottal and laryngeal sites may serve to guard the larynx from invasion by saliva and liquid bolus residues or clear the larynx of secretions wafted up from the trachea and bronchi. Saliva initiates swallowing as readily as water in the cat (4). Laryngeal-receptor function, like visual-receptor function, makes more sense when analyzed in terms of the meaningful stimuli of the environment. Saliva and the remains of a fluid bolus flowing over the epiglottis and glottis are more meaningful than water dropped onto the vocal folds just as a moving fly is more meaningful to the frog's retina than a flashing light (3).

\section{References}

1. Kaнn, R. H. 1903. Studien über den Schluckreflex. I. Die sensible Innervation. Arch. Anat. Physiol. Supp.: 386-426

2. Kawasaki, M., J. H. Ogura, and S. Takenouchi. 1964. Neurophysiologic observations of normal deglutition. Laryngoscope 24 : 1747-1768.

3. Letrvin, J. Y., H. R. Maturana, W. S. McCulloch, and W. H. Pitts. 1959. What the frog's eye tells the frog's brain. Proc. Inst. Radio Eng. 47: 1940-1951.

4. Miller, R. F., and C. S. Sherringiton. 1916. Some observations on the buccopharyigeal slage of reflex deglution in the cat. Quart. J. Exptl. Physiol. 9: 147-186. 
5. Pommerenke, W. T. 1928. A study of the sensory areas eliciting the swallowing reflex. Am. J. Physiol. 84 : 36-41.

6. Prima, G. YA. 1958. "The Role of the Superior Laryngeal Nerve in Regulating Deglutition." A. S. Serafimovich Muncipal Pedogogical Institute, Stalingrad. (Russian)

7. Storey, A. T. 1968. A functional analysis of sensory units innervating epiglottis and larynx. Exptl. Neurol. $20: 366-383$.

8. WassiliefF, N. 1888. Wo wird der Schluckreflex ausgelöst? Z. Biol. 24: 29-47.

9. Waller, A., AND J. L. PRÉvost. 1870. Étude relative aux nerfs sensitifs qui président aux phénomènes réflexes de la déglutition. Arch. Physiol. Norm. Pathol. 3 : 343-354. 